\title{
Pulsar Timing Arrays
}

\author{
Maura McLaughlin \\ West Virginia University \\ email: maura.mclaughlin@mail.wvu.edu
}

\begin{abstract}
I describe the concept of a pulsar timing array and give broad overview of the construction of a pulsar timing array, methods for high-precision timing and noise characterization, and algorithms for gravitational wave detection and source characterization. I then provide an overview of worldwide pulsar timing programs and the scale and sensitivity of the pulsar timing array efforts, with particular attention to the International Pulsar Timing Array (IPTA). I discuss the most recent results from pulsar timing arrays, emphasizing the gravitational wave detection efforts in particular. Finally, I describe the anticipated future growth in participants, telescopes, pulsars, and sensitivity of the IPTA, highlighting the transformational advances that it will enable over the next decade.
\end{abstract}

Keywords. pulsars, pulsar timing, millisecond pulsars, gravitational waves

\section{Introduction}

Millisecond pulsars are incredibly stable celestial clocks that can be used for a variety of fundamental physics experiments. A pulsar timing array is a set of these pulsars can be exploited to search for correlated perturbations in their pulse times of arrival due to gravitational waves. Here, I describe the the current worldwide pulsar timing array efforts, and the observations and analysis necessary to accomplish this goal. The sensitivity of our pulsar timing array experiments is expected to dramatically increase over the next several years, leading to either a gravitational wave detection or very stringent constraints on low-frequency gravitational wave source populations before the end of the decade.

\section{Worldwide Timing Array Efforts}

Pulsars are rapidly rotating, highly magnetized neutron stars produced in the supernova explosions of massive stars. Millisecond pulsars (MSPs) are a subset of these objects which have very short (less than $20 \mathrm{~ms}$ ) rotation periods and phenomenal rotational stability. With the largest radio telescopes in the world, we can measure the arrival times of pulses from these objects very precisely. A pulsar timing array (PTA) is a spatial array of pulsars that is analyzed to detect correlated timing perturbations (Foster \& Backer 1990). There are many science applications for PTAs, including searching for perturbations due to gravitational waves (GWs) with a characteristic quadrupolar angular correlation (Hellings \& Downs 1983; Figure 1).

There are currently three collaborations which are using timing observations of MSPs to search for and characterize GWs. Of the over 2500 pulsars known today, over 300 are MSPs and of these nearly 100 are being timed by PTA projects worldwide, with many MSPs timed by multiple PTAs.

The Parkes Pulsar Timing Array, or PPTA, undertakes observations with the 64-m Parkes Telescope in NSW, Australia at radio frequencies of 0.7, 1.4, and $3 \mathrm{GHz}$ (Manchester et al. 2013). 

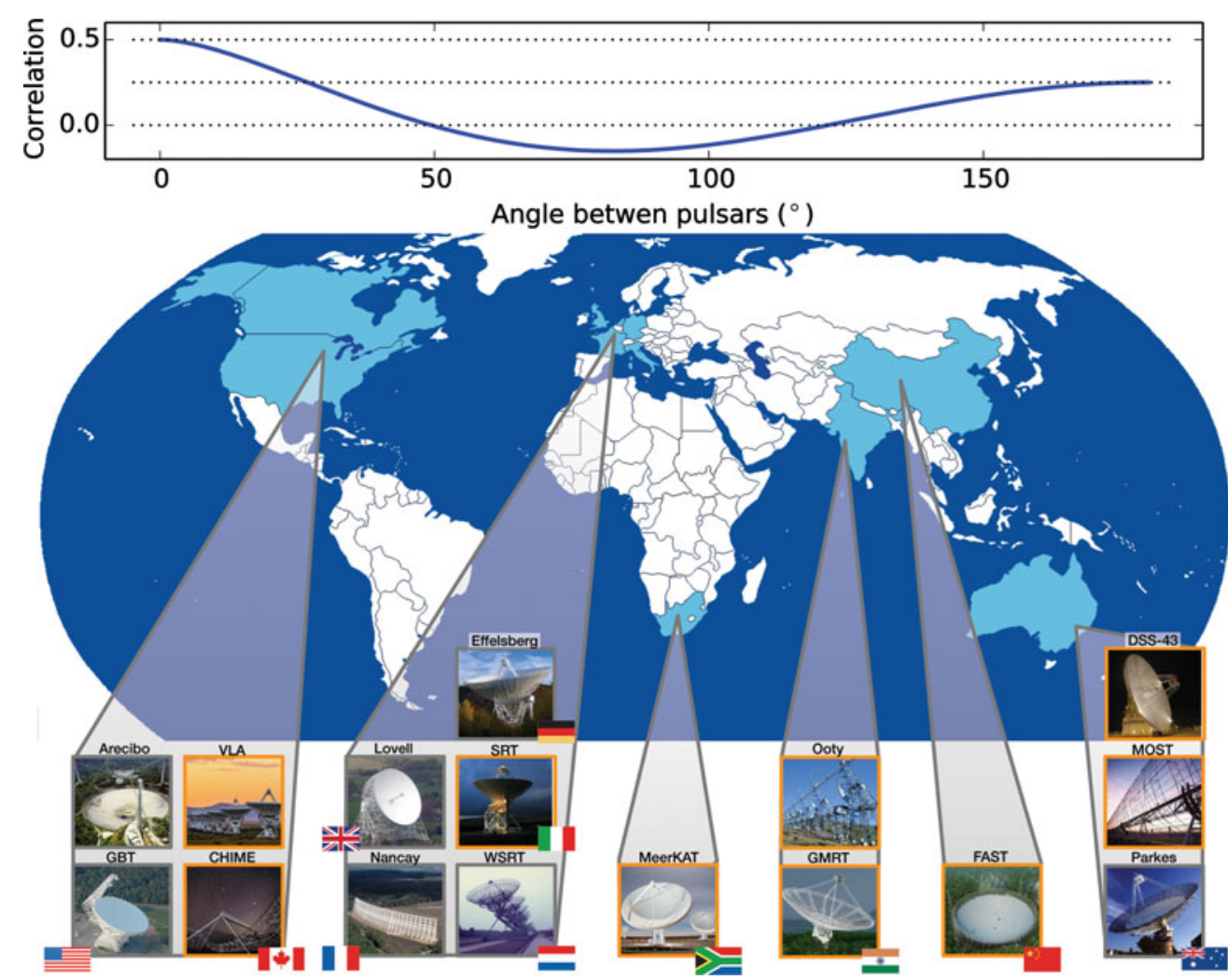

Figure 1. Top: "Hellings and Downs curve" (Hellings \& Downs 1983), the correlation in pulsar timing residuals as a function of MSP angular separation that we expect to detect. Bottom: The telescopes that are or will be used for worldwide pulsar timing experiments. Arecibo (Puerto Rico), the Green Bank Telescope (GBT; West Virginia), Lovell (U.K.), Effelsberg (Germany), Nançay (France), Westerbork Synthesis Radio Telescope (WSRT; Netherlands), Sardinia Radio Telescope (SRT; Italy), and Parkes (Australia) are currently contributing IPTA data. The Very Large Array (VLA; New Mexico) will soon begin to contribute. The Deep Space Station-43 (DSS-43; Australia), the Molonglo Observatory Synthesis Telescope (MOST; Australia), the Giant Meterwave Radio Telescope (GMRT; India) and Ooty (India) as well as three underconstruction telescopes - CHIME (Canada), Five hundred meter Aperture Spherical Telescope (FAST; China), and MeerKAT (South Africa) - will begin contributing pulsar timing data within the next five years. (Image courtesy of S. Chatterjee)

The European Pulsar Timing Array, or EPTA, uses five telescopes in Europe, including the 76-m Lovell Telescope in the UK, the 100-m Effelsberg Telescope in Germany, the 95-m (effective diameter) Nançay Radio Telescope (NRT) in France, the 93-m (effective diameter) Westerbork Synthesis Radio Telescope (WSRT) in the Netherlands, and the newly commissioned 64-m Sardinia Radio Telescope (SRT) in Italy (Kramer \& Champion 2013). The observations are primarily at $1.4 \mathrm{GHz}$, supplemented by low-frequency (350$\mathrm{MHz}$ ) WSRT observations and >2 GHz Effelsberg, NRT, and WSRT observations.

The North American Nanohertz Observatory for Gravitational Waves (NANOGrav) uses the 300-m Arecibo Observatory in Puerto Rico and the 100-m Green Bank Telescope (GBT) in West Virginia, USA at frequencies of 800 and $1.4 \mathrm{GHz}$ with the GBT and at two of 0.4, 1.4, and $2 \mathrm{GHz}$ at Arecibo (McLaughlin 2013).

The PPTA, EPTA, and NANOGrav are all part of the International Pulsar Timing Array (IPTA, Figure 1). While an IPTA data sharing agreement was signed in 2009, 


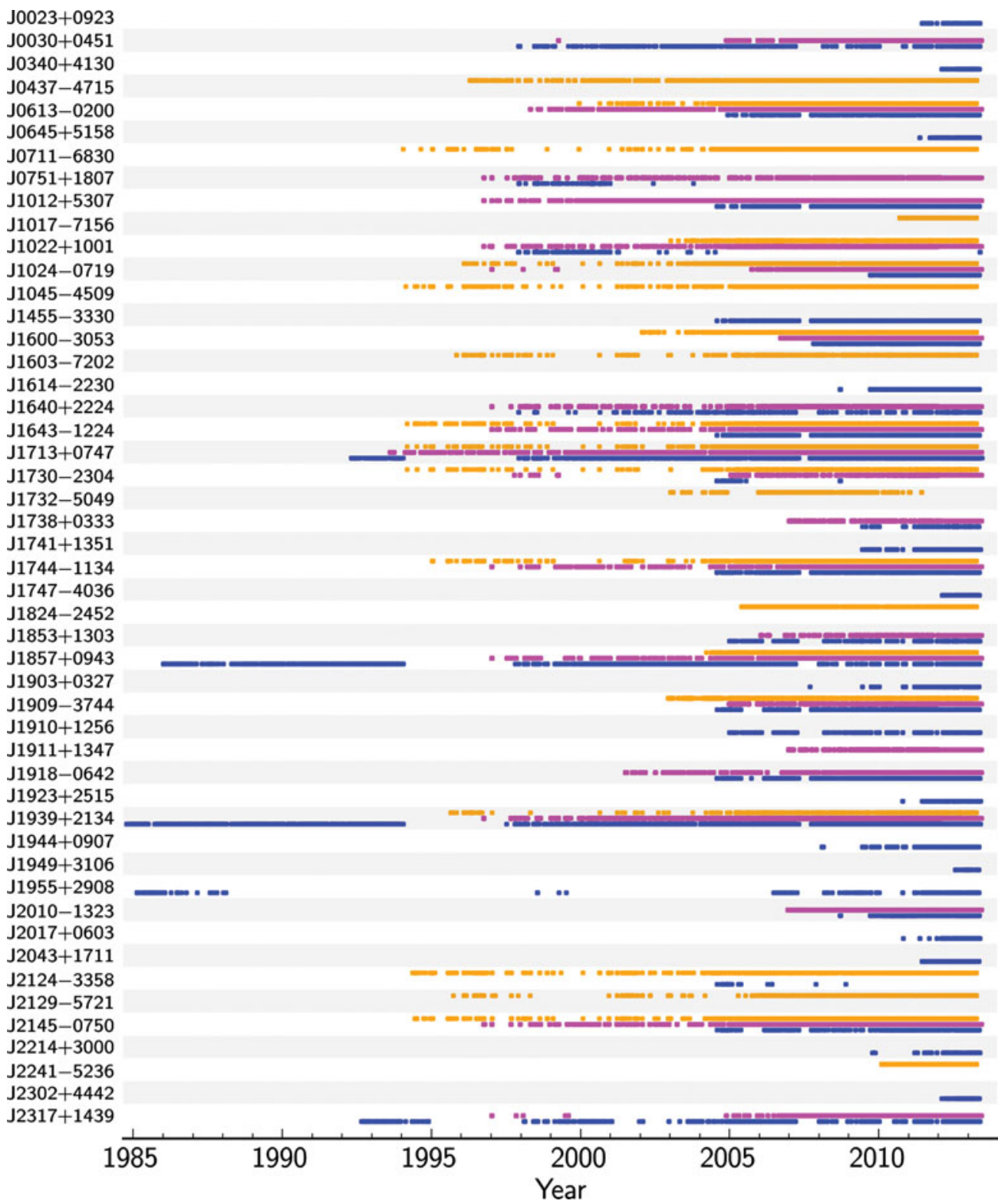

Figure 2. Distribution of TOAs for IPTA data from 1985 to 2014. A total of 187,460 TOAs from 49 MSPs, named on the left, will be available. NANOGrav TOAs are in blue, EPTA TOAs are fuchsia, and PPTA TOAs are orange. (Figure courtesy of R. Lynch)

combining data from all three PTAs and all seven (soon to be eight, with the SRT) telescopes is non-trivial due to clock offsets and varying data formats. The first IPTA data release was recently completed, and the first papers will be published soon (Figure 2; Verbiest et al. 2015, in prep.).

\section{Pulsar Timing Array Data Analysis}

The first step in our GW detection experiment is to measure the times of arrival (or TOAs) of radio pulses from a collection of MSPs as precisely as possible and to fit an 


\section{Input}

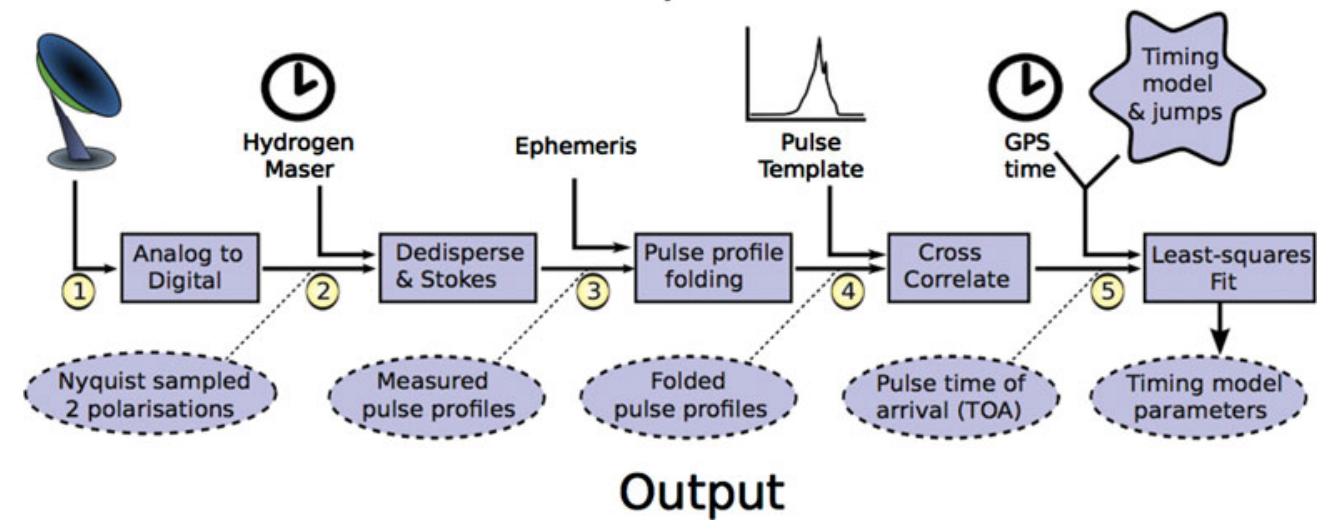

Figure 3. The stages of pulsar timing, beginning with the observation of a pulsar with a radio telescope and resulting in the calculation of a timing model (van Haasteren et al. 2011). In this Figure, adding the Stokes parameters comes before folding, but typically it is done after. (Figure courtesy of R. van Haasteren)

accurate 'timing model' to those TOAs. Figure 3 illustrates the steps involved in this process; a more complete description may be found in Lorimer \& Kramer (2005). A pulsar is first observed with a radio telescope, with signals in two polarizations digitized and recorded. The data are then dedispersed to correct for frequency-dependent delays induced by the ionized free electrons in the interstellar medium. The correction is typically done coherently by convolving the raw signal voltage with the inverse of the interstellar medium transfer function. The data are then folded with an ephemeris containing an MSP's most up-to-date timing model to create full-Stokes profiles across the observation (i.e. one profile every 10 seconds to several minutes) and in a number of frequency subbands (i.e. typically 10 to 500, depending on the width of the radio bandpass). The Stokes parameters are added to create total intensity profiles, using calibration observations to ensure that the parameters are correctly combined. A TOA is measured for each profile by cross-correlating a composite profile (i.e. summed over thousands to millions of individual pulses) with a high signal-to-noise template that is typically the sum of pulse profiles over many epochs. These measured TOAs are then fit to a timing model, incorporating a number of effects both extrinsic and intrinsic to the pulsar, through a least-squares fit (Edwards et al. 2006). Traditionally, DM is fit using TOAs at multiple frequencies along with other parameters. However, new methods that measure a DM and TOA simultaneously demonstrate increased timing precision, in particular when wide bandwidths, over which pulse profiles evolve significantly, are used (Pennucci et al. 2014, Liu et al. 2014). If multiple telescopes or observing backends are jointly fit, as in the first IPTA data release, it may be necessary to incorporate jumps, or phase offsets between sets of TOAs although simultaneous observations with multiple telescopes should result in solving for these parameters and making their inclusion in timing models unnecessary (Dolch et al. 2014).

Once a timing model for an MSP is achieved, "timing residuals" (Figure 4) are calculated by subtracting the model-predicted TOAs from the measured TOAs. The precision with which we can time a pulsar is generally quantified through the root mean square (or RMS) of the timing residuals. There are many factors which determine the achievable RMS for a particular pulsar. To first order, the precision will be proportional to the ratio of the profile's width to its signal-to-noise. Therefore, MSPs with narrow pulse profiles 


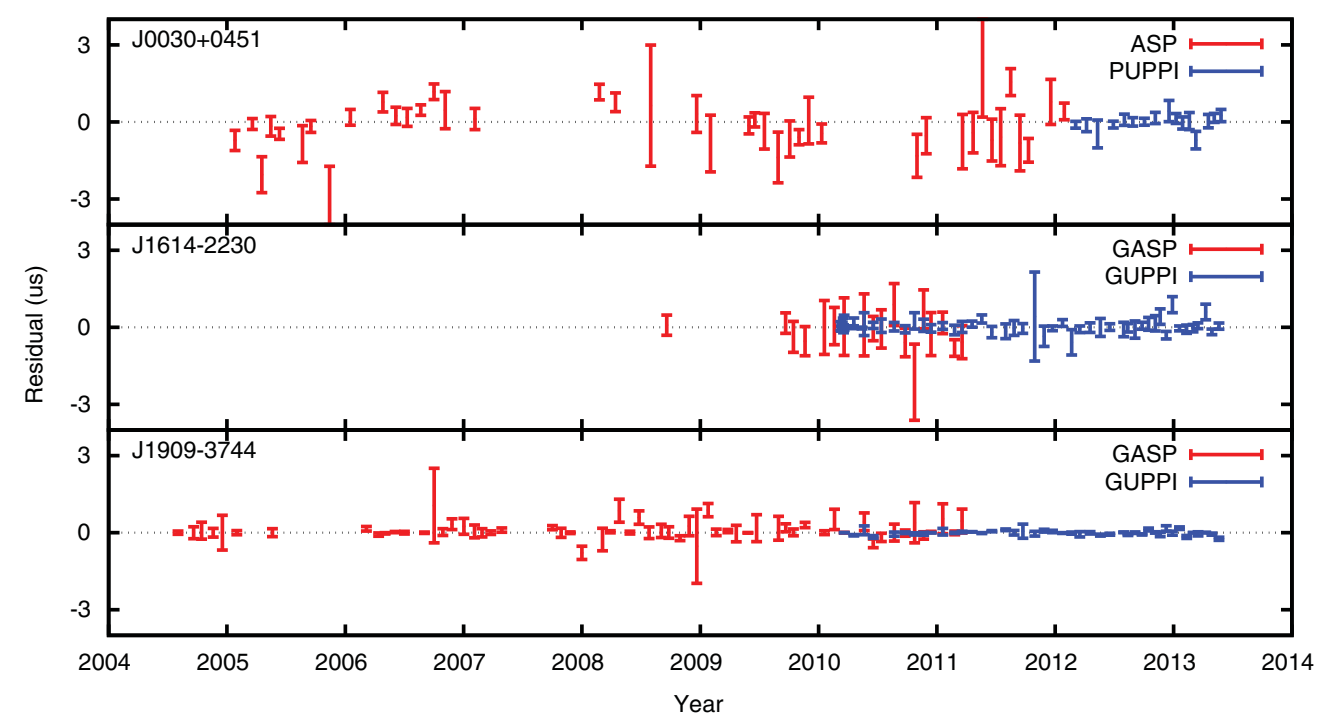

Figure 4. Timing residuals for three MSPs timed by NANOGrav at a radio frequency of $1.4 \mathrm{GHz}$ (Arzoumanian et al. 2015a). Data taken with the ASP/GASP backends, with $64 \mathrm{MHz}$ bandwidth, are shown along with those taken using new backends GUPPI and PUPPI, with $600-800 \mathrm{MHz}$ bandwidths. The increase in bandwidth provides factors of two-three increased timing precision. (Figure courtesy of P. Demorest)

generally have higher timing precisions. The signal-to-noise of the profile will obviously be proportional to the brightness of the MSP at the frequency of observation and also to the observing system's sensitivity, which scales linearly with collecting area and with the square roots of integration time and bandwidth (Lorimer \& Kramer 2005). Therefore, TOA precisions are greater for bright MSPs with narrow pulses observed with large telescopes for long integration times and wider bandwidths.

\section{Gravitational Wave Detection}

Gravitational waves will cause the light travel times between objects along their path to vary, and hence will induce perturbations in the times of arrival of pulsar signals as they travel to Earth. The total time delay induced by a GW source will depend on the GW strain at the pulsar at the time of the radio wave emission (i.e. the 'pulsar term'), the GW strain at the Earth at the time of the radio wave reception (i.e. the 'Earth term'), the orientation of the source, pulsar, and Earth, and the GW frequency, with the induced residual inversely proportional. With this scaling, and an average timing precision of 100 ns over a total timespan of 5-10 years, a PTA would be sensitive to strains of order $10^{-15}$ to $10^{-16}$ (Jenet et al. 2005). This effect will be detected as a characteristic directional signature in the timing residuals with a quadrupolar angular correlation on the sky (Hellings \& Downs 1983; Figure 1), with the exact signature varying depending on the type of GW source. For all cases, GW searches will be performed on timing residuals or by maximizing or marginalizing the likelihood over the timing model parameters in such a way that the timing model parameter measurements and GW wave searches are performed simultaneously.

The sensitivity of a pulsar timing array experiment will peak at GW frequencies corresponding to the inverse of the total timespan of the experiment. For example, the minimum detectable strain for a timing program with a duration of 10 years will be at 




Figure 5. The range of amplitudes, measured by strain $h$, and frequencies to which the four essential GW detection techniques are sensitive are shown, along with the expected sources that would produce GWs at these amplitudes and frequencies. Cosmic microwave background polarization experiments will aim to confirm the B-mode polarization detection. At higher GW frequencies, experiments are targeting astrophysical sources. PTA experiments include NANOGrav, the EPTA, and the PPTA, which together form the IPTA. A space-based interferometer called eLISA (Evolved Laser Interferometer Space Antenna) is currently in the development phase, with a planned European Space Agency launch date of 2032. Ground-based GW interferometers include LIGO (USA), VIRGO (Italy/France), TAMA300 and LCGT (Japan), and GEO600 (Germany/U.K.). The most sensitive of these experiments, LIGO, which consists of two detectors, one in Louisiana and one in Washington, has recently undergone a sensitivity upgrade to become Advanced LIGO.

a frequency of $3 \times 10^{-9} \mathrm{~Hz}$. At frequencies higher than this, the signatures induced in pulsar timing residuals are smaller because of the intrinsic inverse dependence on GW frequency (Detweiler 1979). At frequencies lower than this, the signal would be largely absorbed in timing model fits. In Figure 5, we illustrate how the frequencies at which PTAs are sensitive compare to those of other GW detection experiments.

\section{Current Stochastic Background Limits}

All three PTA collaborations are performing several different kinds of GW analyses that are sensitive to different types of GW sources. These include stochastic, burst, and continuous sources. The stochastic background detectable by PTAs likely includes contributions from supermassive black hole binaries (Sesana 2013) and, possibly, cosmic strings (Siemens et al. 2006), early Universe phase transitions (Caprini et al. 2010), and relic GWs from inflation (Tong et al. 2014). Continuous GW sources emit at a single (but evolving) GW frequency, with supermassive black hole binaries the most likely source of continuous GWs for PTAs. Burst sources have signal durations much shorter than the total time spans of observations and could be due to mergers of SMBHs, periastron passages of compact objects orbiting a SMBH, or cusps on cosmic strings (Damour \& Vilenkin 2005). 


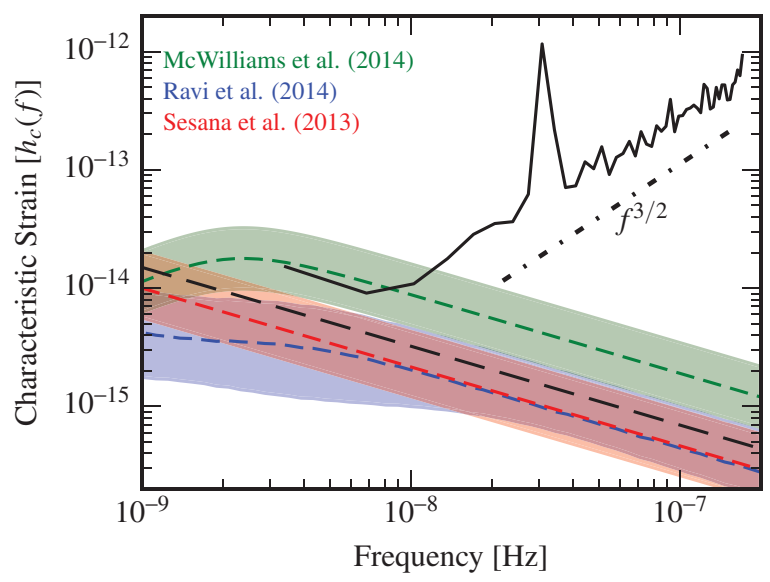

Figure 6. Strain amplitude vs. GW frequency. The solid black and long dashed black lines are the $95 \%$ upper limits from the spectral and power-law analyses of Arzoumanian et al. (2015b). The red, blue and green shaded regions are the one-sigma predictions from the models of Sesana (2013), Ravi et al. (2014), and McWilliams et al. (2014). The green shaded region uses the simulation results from McWilliams et al. (2014), but replaces the fit to the GWB predictions used in that paper with a model with a spectral turnover. From Arzoumanian et al. (2015b), reproduced by permission of the AAS.

In order to compare the three PTA efforts and gauge the overall sensitivity of the worldwide search for low-frequency GWs, we review the upper limits placed by the three PTAs on the GW stochastic background. The most recent 95\% confidence upper limits on the characteristic strain $h_{c}$ at a frequency of $1 \mathrm{yr}^{-1}$ are $1 \times 10^{-15}, 6 \times 10^{-15}$, and $1.5 \times 10^{-15}$ for the PPTA, EPTA, and NANOGrav, respectively (Shannon et al. 2015; van Haasteren et al. 2011; Arzoumanian et al. 2015b). These limits are based on datasets of length 11, 8, and 9 years, respectively. All assume that the stochastic GW background has a spectral slope of $-2 / 3$. The PPTA limit was calculated from the power spectra of the four PPTA pulsars with the highest timing precisions, while both of the EPTA and NANOGrav limits were based on analyses that constrain the presence of the expected angular correlation due ue to a GW background using five pulsars (EPTA) and 37 pulsars (NANOGrav). These limits are setting meaningful constraints on astrophysical models for supermassive black hole binary growth and evolution (Figure 6).

\section{The Future of Gravitational Wave Detection with PTAs}

The primary goal of the PPTA, EPTA, NANOGrav, and of the broader IPTA consortium over the past several years has been building and characterizing a sensitive GW detector. This work has involved searching for MSPs with large radio telescopes, characterizing the properties of the MSPs, and determining of the optimal techniques for taking processing, storing, and sharing timing data. Once PTAs begin to make their first detections, the focus will shift from building and characterization to $G W$ source characterization. Given our current understanding of source populations and the current and projected sensitivities of the IPTA, we expect this shift to happen within the next decade and possibly within the next several years. Measurements of the amplitude and spectrum of the stochastic background of GWs at the low frequencies at which PTAs are sensitive will enable constraints on source populations and will have significant impacts on the development of more accurate models for the formation and evolution of galaxies. It will also allow sensitive tests of general relativity that have never before been possible. The 
detection of continuous wave and burst GW sources will allow characterization of individual supermassive black hole binaries that could be accompanied by electromagnetic observations, facilitating multi-messenger studies of these sources. In the future, with the advent of telescopes such as FAST, MeerKAT, and, ultimately, the Square Kilometre Array (SKA), hundreds of high-precision MSPs will be timed, allowing for the detection and characterization of many GW sources, leading to a true Galactic-scale GW observatory.

\section{References}

Arzoumanian, Z., et al.: The NANOGrav Collaboration 2015, ApJ, in press; arXiv:1505.07540 Arzoumanian, Z.: The NANOGrav Collaboration 2015, ApJ, in press; arXiv:1508.03024

Caprini, C., Durrer, R., \& Siemens, X. 2010, Phys. Rev. D, D82, 063511

Damour, T. \& Vilenkin, A. 2005, Phys. Rev. D, 71, 063510

Detweiler, S. 1979, ApJ, 234, 1100

Dolch, T., Lam, M. T., Cordes, J. M., et al. 2014, ApJ,794, 21

Edwards, R. T., Hobbs, G. B., \& Manchester, R. N. 2006, MNRAS, 372, 1549

Foster, R. S. \& Backer, D. C. 1990, ApJ, 361, 300

Hellings, R. W. \& Downs, G. S. 1983, ApJ, 265, L39

Jenet, F. A., Hobbs, G. B., Lee, K. J., \& Manchester, R. N., ApJ, 625, L123

Kramer, M. \& Champion, D. J. 2013, Classical Quant. Grav., 30, 224009

Liu, K., Desvignes, G., Cognard, I., et al. 2014, MNRAS, 443, 3752

Lorimer, D. R. \& Kramer, M. 2005, Handbook of Pulsar Astronomy (Cambridge Univ. Press: Cambridge)

Manchester, R. N., Hobbs, G., Bailes, M., et al. 2013, PASP, 30, 17

McLaughlin, M. A. 2013, Classical Quant. Grav., 30, 224008

McWilliams, S. T., Ostriker, J. P., \& Pretorius, F. 2014, ApJ, 789, 156

Pennucci, T. T., Demorest, P. B., \& Ransom, S. M. 2014, ApJ, 790, 93

Ravi, V., Wyithe, J. S. B., Shannon, R. M., Hobbs, G., \& Manchester, R. N. 2014, MNRAS, 442,56

Sesana, A. 2013, MNRAS, 433, L1

Siemens, X., Creighton, J., Maor, I., et al. 2006, Phys. Rev. D, 73, 105001

Shannon, R. M., Ravi, V., Lentati, L. T., et al. 2015, Science, 349, 1522

Tong, M. L., Zhang, Y., Zhao, W., et al. 2014, Classical Quant. Grav., 31, 035001

van Haasteren, R., Levin, Y., Janssen, G. H., et al. 2011, MNRAS, 414, 3117 